\title{
SOME PROPERTIES OF ORDINAL DIAGRAMS
}

\author{
MARIKO YASUGI*
}

The theory of ordinal diagrams has been a most powerful means for consistency proofs of some systems of second order arithmetic. The last existing result in this line is the consistency proofs of the systems with the provably $\Delta_{2}^{1}$-comprehension axiom and the $\Delta_{2}^{1}$-comprehension axiom respectively (cf. [6]). In order to pursue the consistency problem further, one needs investigate the theory of ordinal diagrams in two directions-refinement and strengthening of the theory.

For this purpose we have begun to search for some properties concerning ordinal diagrams and some variations of the theory of ordinal diagrams. The reader is requested to refer to $\S 26$ of [7] for the basic knowledge of ordinal diagrams.

\section{§1. Comparison of orderings}

Let $\boldsymbol{O}(\boldsymbol{I}, \boldsymbol{A})$ be a system of ordinal diagrams (o.d.'s), let $\tilde{\boldsymbol{I}}$ be $\boldsymbol{I} \cup\{\infty\}$ and let $i$ be an element of $\tilde{\boldsymbol{I}}$ (namely an indicator or $\infty$ ). Here we shall compare the order-types of the orderings $\angle_{i}$ for $\boldsymbol{O}(\boldsymbol{I}, \boldsymbol{A})$ for distinct $i$ 's.

LEMMA 1. Let $i$ and $j$ be elements of $\tilde{\boldsymbol{I}}$ where $i<j$. Then $\angle_{i}$ can be embedded in $\angle{ }_{j}$.

Proof. Fix $a$ an element of $A$, and for every $\alpha$ (of $\boldsymbol{O}(\boldsymbol{I}, \boldsymbol{A})$ ) define an assignment of connected o.d. $\tilde{\alpha}=(i, a, \alpha)$. This assignment defines a one-to-one mapping of $\boldsymbol{O}(\boldsymbol{I}, \boldsymbol{A})$ onto $\boldsymbol{O}[i, a]$, where

$$
\boldsymbol{O}[i, a]=\{(i, a, \alpha) ; \alpha \in \boldsymbol{O}(\boldsymbol{I}, \boldsymbol{A})\} .
$$

Obviously $\boldsymbol{O}[i, a]$ is a proper subset of $\boldsymbol{O}(\boldsymbol{I}, \boldsymbol{A}), \tilde{\alpha} \angle_{j} \tilde{\beta}$ if and only if $\alpha{ }_{i} \beta$ since $j>i$. Thus, the mapping is order-preserving from

Received March 7, 1977.

* This work has been completed during my stay at the Research Institute for Mathematical Sciences in Kyoto. 
$\left(\boldsymbol{O}(\boldsymbol{I}, \boldsymbol{A}), \angle_{i}\right)$ into $\left(\boldsymbol{O}(\boldsymbol{I}, \boldsymbol{A}), \angle_{j}\right)$.

Proposition 1.1. Let $i$ and $j$ be indicators where $i<j$. Then $\angle_{i}$ is embeddable in a proper, initial segment of $\angle_{j}$.

Proof. Take 0 (the initial element of $A$ ) as the $a$ in Lemma 1.

$$
(i, 0, \alpha) \angle_{j}(j, 0,0)
$$

for every $\alpha$. So $\angle_{i}$ is embedded in $\angle_{j} \Gamma(j, 0,0)$ where, in general,

$$
\angle \Gamma \alpha=(\{\beta ; \beta<\alpha\}, \angle) \text {. }
$$

Proposition 1.2. If $i$ is an indicator and if there is a value $(k, a)$ which is greater than $(i, 0)$, then $\angle_{i}$ is embeddable in a proper, initial segment of $\angle_{\infty}$.

Proof. $\quad(i, 0, \alpha) \angle_{\infty}(k, a, 0)$.

LEMMA 2. Let $j$ be an indicator. Let $\tilde{L}_{j}$ denote the ordering $\angle_{1}$ restricted to

$$
\boldsymbol{O c}(j)=\{\beta ; \beta \text { is connected and } \beta \angle,(j, 0,0)\} .
$$

Then

$$
\left|\tilde{Z}_{j}\right|=\sum_{i<j}\left|\angle_{i}\right| \times|A|,
$$

where $|\angle|$ denotes the order type of $\angle,|A|$ denotes the order type of $\angle_{A}$, and $\sum_{i<j} \mu_{i}$ is the infinite sum of the ordinals $\mu_{i}$ defined along the order of $\boldsymbol{I}$.

Proof. (1) $\left(\boldsymbol{O}(\boldsymbol{I}, \boldsymbol{A}), \angle_{i}\right) \simeq\left(\boldsymbol{O}[i, a], \angle_{j}\right)$ (isomorphic) for each $a$ in $A$ if $i<j$ (Lemma 1 ).

(2) $\boldsymbol{O c}(j)=\cup_{i<j}\{(i, a, \alpha) ; \alpha \in A$ and $\alpha \in \boldsymbol{O}(\boldsymbol{I}, \boldsymbol{A})\}=\cup_{i<j} \cup_{a \in A} \boldsymbol{O}[i, \alpha]$.

(3) $(i, a, \alpha){ }_{j}(i, b, \beta)$ if $i<j$ and $a<b$.

(4) $(i, a, \alpha){ }_{j}(k, b, \beta)$ if $i<k<j$.

(1) through (4) yield the desired equation.

LEMMA 3. Let $\tilde{L}_{\infty}$ denote the ordering $\angle_{\infty}$ restricted to connected o.d.'s. Then

$$
\left|\tilde{L}_{\infty}\right|=\sum_{i \in I}|\angle i| \times|A| \text {. }
$$

From Lemmas 1 and 2 follows immediately; 
THEOREM 1. Let $\angle_{j}^{*}$ be $\angle_{j}$ restricted to the elements below $(j, 0,0)$ with respect to $\angle_{j}$ if $j$ is an indicator, and let $\angle_{j}^{*}$ be $\angle_{j}$ if $j$ is $\infty$. For any $j$,

$$
\left|\angle_{j}^{*}\right|=\omega^{\sum_{i<j}|<i| \times|\boldsymbol{A}|}
$$

\section{§2. Limit properties}

Here we shall investigate some ordinals which have certain limit properties with regards to ordinal diagrams.

Proposition 2.1. Let 0 denote the least element of $I$. The least ordinal $\Omega$ such that

$$
\forall \alpha<\Omega\left(|\boldsymbol{O}(\boldsymbol{I}, \alpha \times \boldsymbol{A})|_{\angle 0}<\Omega\right)
$$

is obtained as follows. ( $\alpha \times \boldsymbol{A}$ is ordered lexicographically and $|\boldsymbol{B}|_{\llcorner}$denotes the order type of $\angle$ when it is an order of $\boldsymbol{B}$.)

Define $\boldsymbol{O}_{i}$ and $\angle^{i}$ for $i<\omega$ :

$\boldsymbol{O}_{0}=\boldsymbol{O}(\boldsymbol{I}, \boldsymbol{A})$ and $\angle^{0}$ is the order $\angle_{0}$ for $\boldsymbol{O}_{0} ; \boldsymbol{O}_{i+1}=\boldsymbol{O}\left(\boldsymbol{I}, \boldsymbol{O}_{i} \times \boldsymbol{A}\right)$, where $\boldsymbol{O}_{i} \times \boldsymbol{A}$ is given the lexicographical order, $\boldsymbol{O}_{i}$ with the order $\angle^{i}$ and $\angle^{i+1}$ is the order $\angle_{0}$ for $\boldsymbol{O}_{i+1}$. Define

$$
\Omega=\sup _{i<\omega}\left|\angle^{i}\right|
$$

This $\Omega$ will do.

Proof. (1) $\left\{\left|\angle^{i}\right|\right\}_{i}$ is an increasing sequence of ordinals.

(2) $\forall \alpha<\Omega \exists i\left(\alpha \leq\left|\angle^{i}\right|\right)$

Suppose $\Omega$ does not satisfy the condition $(*)$. Then

(3) for some $\alpha<\Omega,|\boldsymbol{O}(\boldsymbol{I}, \alpha \times A)|_{\angle_{0}} \geq \Omega$.

On the other hand, (2) implies that there is an $i$ such that

(4) $\alpha \leq\left|\angle^{i}\right|$.

From (3) and (4) follows

$$
\Omega \leq|\boldsymbol{O}(\boldsymbol{I}, \alpha \times \boldsymbol{A})|_{\angle_{0}} \leqslant\left|\boldsymbol{O}\left(\boldsymbol{I}, \boldsymbol{O}_{i} \times \boldsymbol{A}\right)\right|_{\angle_{0}}=\left|\angle^{i+1}\right|,
$$

contradicting the definition of $\Omega$. Thus $\Omega$ satisfies $(*)$.

Suppose next there is another $\Omega^{\prime}$ such that $(*)$ holds for $\Omega^{\prime}$. Taking $\alpha$ to be 1 , we have

(5) $\left|\angle{ }^{0}\right|<\Omega^{\prime}$.

(6) Assume $\left|\angle^{i}\right|<\Omega^{\prime}$. Taking $\alpha$ to be $\boldsymbol{O}_{i}$ with the order $\angle^{i}$, we 
obtain $\left|\boldsymbol{O}_{i+1}\right|_{\angle_{0}}<\Omega^{\prime}$, or $\left|\angle^{i+1}\right|<\Omega^{\prime}$.

From (5) and (6) we can conclude that $\Omega$ is the least ordinal satisfying $(*)$.

Proposition 2.2. The $\Omega$ in Proposition 2.1 is primitive recursive, presuming that the orderings of $I$ and $A$ are.

Proof. (1) Define $\tilde{\boldsymbol{O}}_{i}$ by

$$
\tilde{\boldsymbol{O}}_{i}=\left\{(i ; \alpha) ; \alpha \in \tilde{\boldsymbol{O}}_{i}\right\}
$$

and $\tilde{\boldsymbol{O}}$ by

$$
\tilde{\boldsymbol{O}}=\bigcup_{i<\omega} \tilde{\boldsymbol{O}}_{i}
$$

Define an ordering $\tilde{Z}$ for $\tilde{\boldsymbol{O}}$ :

$(i ; \alpha) \tilde{Z}(j ; \beta)$ if and only if $i<j$ or $i=j \wedge \alpha \angle^{i} \beta . \quad \tilde{Z}$ is primitive recursive.

(2) Define $O_{n}^{*}$ as follows.

$$
\boldsymbol{O}_{0}^{*}=\tilde{\boldsymbol{O}}_{0} ; \boldsymbol{O}_{n+1}^{*}=\boldsymbol{O}_{n}^{*} \cup \tilde{\boldsymbol{O}}_{n+1} .
$$

Define ${ }^{n} \angle$ for $\boldsymbol{O}_{n}^{*}$ to be the order $\tilde{\angle}$ restricted to $\boldsymbol{O}_{n}^{*}$.

(3) $\left|{ }^{n} \angle\right|=\left|\angle^{n}\right|$ for every $n<\omega$.

This is proved by induction on $n$. Assume $\left.\right|^{n} \angle|=| \angle{ }^{n} \mid$. Then $\left.\right|^{n} \angle|<| \angle^{n+1} \mid$, hence

$$
\left.\left.\right|^{n+1} \angle|=| \boldsymbol{O}_{n}^{*} \cup \tilde{\boldsymbol{O}}_{n+1}\right|_{n+1} \angle=\left.\right|^{n} \angle|+| \angle^{n+1}|=| \angle^{n+1} \mid,
$$

since those order types are limit ordinals.

(4) $|\tilde{Z}|=\left.\sup _{n<\omega}\right|^{n} \angle 1$.

$|\tilde{\angle}|=\Omega$ follows from (3) and (4), and by (1) $\Omega$ is primitive recursive.

The following proposition is proved in a like manner.

Proposition 2.3. The least ordinal $\Omega$ which satisfies

(*) $\quad \forall \alpha<\Omega\left(\alpha>0 \supset|\boldsymbol{O}(\boldsymbol{I}, \alpha)|_{L_{0}}<\Omega \wedge|\boldsymbol{O}(\boldsymbol{I}, \boldsymbol{A})|_{L_{0}}<\Omega\right)$

is obtained as follows.

Define $\boldsymbol{O}_{i}$ and $\angle^{i}$, $i<\omega$ :

$\boldsymbol{O}_{0}=\boldsymbol{O}(\boldsymbol{I}, \boldsymbol{A})$ and $\angle{ }^{0}$ is the order $\angle_{0}$ for $\boldsymbol{O}(\boldsymbol{I}, \boldsymbol{A}) ; \boldsymbol{O}_{i+1}=\boldsymbol{O}\left(\boldsymbol{I}, \boldsymbol{O}_{i}\right)$ and $\angle^{i+1}$ is the order $\angle 0$ for $\boldsymbol{O}_{i+1}$. Define $\Omega=\sup _{i<\omega}\left|\angle^{i}\right|$.

Furthermore there is a primitive recursive order for such $\Omega$. 
Proposition 2.4. Let $\left\{\boldsymbol{I}_{n}\right\}_{n}$ be an increasing sequence of well-ordered sets; namely $\boldsymbol{I}_{n}$ is embeddable in $\boldsymbol{I}_{n+1}$. Let $\boldsymbol{I}$ be $\lim \boldsymbol{I}_{n}$, or $\boldsymbol{I}=\bigcup_{n} \boldsymbol{I}_{n}$ with the order induced from those of $\left\{\boldsymbol{I}_{n}\right\}_{n}$. Assume the same for $\left\{\boldsymbol{A}_{n}\right\}_{n}$ and A. Then

$$
\lim _{n}\left|\boldsymbol{O}\left(\boldsymbol{I}_{n}, \boldsymbol{A}_{n}\right)\right|_{L_{0}}=|\boldsymbol{O}(\boldsymbol{I}, \boldsymbol{A})|_{L_{0}} .
$$

Proof. (1) $\boldsymbol{O}\left(\boldsymbol{I}_{n}, \boldsymbol{A}_{n}\right) \subset \boldsymbol{O}\left(\boldsymbol{I}_{m}, \boldsymbol{A}_{m}\right)$ if $n<m$ and $\cup_{n} \boldsymbol{O}\left(\boldsymbol{I}_{n}, \boldsymbol{A}_{n}\right)=\boldsymbol{O}(\boldsymbol{I}, \boldsymbol{A})$ as sets.

(2) For every $i$ in $I_{n},\left(\boldsymbol{O}\left(\boldsymbol{I}_{n}, \boldsymbol{A}_{n}\right), \angle_{i}\right)$ is embedded in $\left(\boldsymbol{O}\left(\boldsymbol{I}_{m}, \boldsymbol{A}_{m}\right), \angle_{i}\right)$ if $n<m$.

(3) $\left(\boldsymbol{O}\left(\boldsymbol{I}_{n}, \boldsymbol{A}_{n}\right), \angle_{i}\right)$ is embedded in $\left(\boldsymbol{O}(\boldsymbol{I}, \boldsymbol{A}), \angle_{i}\right)$ for every $i$ in $\boldsymbol{I}_{n}$ and every $n$.

(4) $\left\{\left(\boldsymbol{O}\left(\boldsymbol{I}_{n}, \boldsymbol{A}_{n}\right), \angle_{0}\right)\right\}_{n}$ is an increasing sequence of well-ordered sets. (cf. (2) above.)

(5) (2) (4) imply that

$$
\bigcup_{n}\left(\boldsymbol{O}\left(\boldsymbol{I}_{n}, A_{n}\right), \angle_{0}\right)=\left(\boldsymbol{O}(\boldsymbol{I}, \boldsymbol{A}), \angle_{0}\right),
$$

or

$$
\lim _{n}\left|\boldsymbol{O}\left(\boldsymbol{I}_{n}, \boldsymbol{A}_{n}\right)\right|_{L_{0}}=|\boldsymbol{O}(\boldsymbol{I}, \boldsymbol{A})|_{L_{0}} .
$$

As a special case of Proposition 2.4 we have

Theorem 2. Define $\boldsymbol{O}_{n}$ as follows.

$\boldsymbol{O}_{0}=\boldsymbol{O}(\boldsymbol{I}, \boldsymbol{A}) ; \boldsymbol{O}_{n+1}=\boldsymbol{O}\left(\boldsymbol{O}_{n}, \boldsymbol{O}_{n}\right)$, where $\boldsymbol{O}_{n}$ is ordered by $\angle_{0}$. Then

$$
\Omega=\lim _{n}\left|\boldsymbol{O}_{n}\right|_{L_{0}}=|\boldsymbol{O}(\Omega, \Omega)|_{L_{0}} .
$$

Thus $\Omega$ defined as this is a fixed point for the theory of ordinary diagrams.

\section{§3. A generalization of ordinal diagrams}

By introducing a third basic (well-ordered) set, $A$, one can either refine or strengthen the theory of ordinal diagrams. The elements of $\Lambda$ act as classifiers, namely classifying the power of values.

The theory defined in this section is a straightforward generalization of the original one in [7]. The theory of approximations can be developed as before; only in a more uniform manner (without the irregularity for the first values). 
Definition 3.1. The elements of $\boldsymbol{O}(\Lambda, I, A)$ are defined in a manner similar to those of $\boldsymbol{O}(\boldsymbol{I}, \boldsymbol{A})$; if $(\lambda, i, a)$ is in $\Lambda \times \boldsymbol{I} \times \boldsymbol{A}$ and if $\alpha$ is an element of $\boldsymbol{O}(\Lambda, I, A)$, then $(\lambda, i, a, \alpha)$ is an element of $\boldsymbol{O}(\Lambda, I, A)$. The elements of $\Lambda$ are called classifiers.

Sections are defined for indicators and orderings are defined for the elements of $\tilde{I}$; the classifiers are disregarded for those matter. For example, the $i$-section of $(\lambda, i, a, \alpha)$ is $\alpha$.

$(\lambda, i, a, \alpha) \angle_{\infty}(\mu, j, b, \beta)$ if and only if

$$
(\lambda, j, a)<(\mu, j, b) \text { or }(\lambda, i, a)=(\mu, j, b) \wedge \alpha L_{i} \beta,
$$

where $\Lambda \times I \times A$ are lexicographically ordered.

For an indicator $i, \angle_{i}$ is defined as usual for connected elements. For non-connected elements, the orderings are defined as before.

We omit all the proofs that can be carried out as in [7].

Proposition 3.1. $O(\Lambda, I, A)$ as defined above is linearly ordered for every $\angle_{i}$.

LEMMA 1. 1) If $\sigma$ is an i-section of $\alpha$, then $\sigma \angle_{i} \alpha$. 2) If $\sigma$ is connected and $\beta$ is a proper $i$-subsection of $\alpha$, then $\beta L_{1} \alpha$ for every $j \leq i$.

Proposition 3.2. $\boldsymbol{O}(\Lambda, I, A)$ is well-ordered by $\angle_{i}$ for every $i$ in $\tilde{\boldsymbol{I}}$.

Proposition 3.3. $\left(\boldsymbol{O}(\boldsymbol{I}, \boldsymbol{A}), \angle_{i}\right)$ is embeddable in $\left(\boldsymbol{O}(\Lambda, \boldsymbol{I}, \boldsymbol{A}), \angle_{i}\right)$ for every $i$; or $\left(\boldsymbol{O}(\boldsymbol{I}, \boldsymbol{A}), \angle_{i}\right)$ is isomorphic to $\left(\boldsymbol{O}(\Lambda, \boldsymbol{I}, \boldsymbol{A}), \angle_{i}\right)$ when $\Lambda$ is a singleton.

THEOREM 3. The theory of approximations can be developed for $\boldsymbol{O}(\Lambda, I, \boldsymbol{A})$.

To develop the theory of approximations, we shall quote the numberings of [7] with*: Def. 26. 41* means Definition 26. 41 in [7], and remark where to make adjustments. We shall, however, omit obvious alterations.

The "values" in the definitions in $\S 26^{*}$ are here of the form $(\lambda, i, a)$.

DEF. 26. $41^{*}$. (1) when $(\lambda, i, a)$ is the outermost value of a connected o.d., $\lambda$ is the outermost classifier and $i$ is the outermost indicator.

(2) Kernels are defined as original; only the indicators matter.

Proof of Prop. 26. 43*. Let $v_{0}(j, \alpha)$ be $(\lambda, i, a)$. (1) $\eta$ is 0 or of 
the form $\left(\mu, k, b, \eta^{\prime}\right)$ where $k<j$. Since $\eta$ is $j$-active in $\beta$ and $v_{0}(j, \beta)$ $<(\lambda, i, a),(\mu, k, b)<(\lambda, i, a)$. Thus $\eta \angle_{m}(\lambda, i, a, \gamma)$ if $j \leq m \leq \infty$ and if $(\lambda, i, \alpha, \gamma)$ is $j$-active in $\alpha$.

DEF. 26. 44*. (1) Let $(\lambda, i, a)=v_{0}(j, \alpha)$. Define the 0 -th $j$-approximation of $\alpha$ as original, replacing $(i, \alpha)$ by $(\lambda, i, a)$.

Proof of (3) of Lemma 26. 45*. Let $\eta$ be $\left(\mu, k, b, \eta^{\prime}\right)$ where $k<j$. Let $\alpha_{0}$ be $\left(\lambda, i, a, \alpha^{\prime}\right) . \quad(\mu, k, b) \leq(\lambda, i, \alpha) . \quad \eta \angle_{\infty} \alpha$ is obvious by virtue of the condition on $\alpha_{0}$. For an $m$ such that $j \leq m<\infty$, there is no $m$ section of $\eta$, hence $\eta \angle_{m} \alpha_{0}$.

Proof of (4) of Lemma 26. 45*. (i) reads at it stands.

(ii) $\eta=\left(\mu, k, b, \eta^{\prime}\right), k \geq j$. If $(\mu, k, b)=(\lambda, i, a)$, then $\eta \angle_{m} \alpha_{0}$ for all $m \geq i$. If $j \geq i$, this will do. $j<i$ and $j \leq m<i$, then use the induction hypothesis. Suppose $(\mu, k, b)<(\lambda, i, a)$. Then $\eta \angle_{m} \alpha_{0}$ for all $m>k$. For an $m, j \leq m \leq k$, use the induction hypothesis.

Remark. (5) of Lemma 26. 45* does not hold for this system as shown below. This is, however, an isolated proposition, and hence it will not affect the main story of the theory of approximations.

(5) Let $v_{0}(j, \alpha)$ be $(\lambda, i, \alpha)$ and let $\delta_{1}, \cdots, \delta_{m}$ be all the $j$-subsections of $\alpha$. Then

$$
\alpha_{0}=\operatorname{apr}(0, j, \alpha)=\max _{\angle i+1}\left(\delta_{1}, \delta_{2}, \cdots, \delta_{m}\right) \text {. }
$$

Counter-example. Consider $\boldsymbol{O}(2,2,2), j=0$ and $\alpha_{0}=(1,0,0,0)$, hence $\lambda=1, i=0, a=0, \alpha^{\prime}=0$ and $\alpha=\left(0,1,0, \alpha_{0}\right) . \quad v_{0}(j, \alpha)=(1,0,0)$. But $\alpha_{0} \angle{ }_{1} \alpha$ where $1=i+1$.

Prop. 26. 47*. Let $\eta$ be a j-subsection of $\alpha$ that contains an $\alpha_{0}$ and let $\alpha_{0}^{1}, \alpha_{0}^{2}, \cdots, \alpha_{0}^{m}$ be all occurrences of $\alpha_{0}$ (as the 0-th $j$-approximation) in $\eta$. Let $q_{k}$ be the least indicator in $\eta$ connected to $\alpha_{0}^{k}, k=1,2, \cdots, m$, and let $q=q(\eta)=\max \left(q_{1}, \cdots, q_{m}\right)$. Then $\eta \angle_{p} \alpha_{0}$ for every $p>q$.

Proof. The original proof goes through; eliminate the condition $p \leq i$.

The irregularity for the first valuation and the first approximation in the original theory is absent in our case; in fact that irregularity is only a consequence of the subsequent, uniform treatment when $I \times A$ is lexicographically ordered for $\boldsymbol{O}(\boldsymbol{I}, \boldsymbol{A})$. Thus we can omit Def. 26. $48^{*} \sim$ Prop. 26. 52*. 
DEF. 26. 53*. Let $i_{0}$ be the indicator in $v_{0}(j, \alpha)$. $\left(\alpha_{0}, i_{0}\right),\left(\alpha_{1}, i_{1}\right)$, $\cdots,\left(\alpha_{n}, i_{n}\right)$ are defined as before, reading $(\mu, k, b, \gamma)$ in the place of $(k, b, \gamma)$. Note that $j \leq i_{m+1}<i_{m}$ if $m \geq 1$, but nothing can be said of $i_{0}$.

The rest of the material, viz., Cor. 26. $54^{*} \sim$ Prop. 26. $61^{*}$, goes through with some minor notational changes.

Remark. 1) The theory $\boldsymbol{O}(\Lambda, \boldsymbol{I}, \boldsymbol{A})$ is a natural generalization of the one in [7], which is isomorphic to $O(1, I, A)$. If $I \times A$ is antilexicographically ordered, then $\boldsymbol{O}(\boldsymbol{I}, A)$ is isomorphic to $\boldsymbol{O}(A, I, 1)$. This is worth noting in connection with the fact that in the existing consistency proofs (cf. [3], [4], [5], [6]), the order of $(i, a)$ is immaterial.

2) $\boldsymbol{O}(\Lambda, I, A)$ can be extended to any basic (well-ordered) sets $\boldsymbol{B}_{1}$, $\cdots, \boldsymbol{B}_{m}$, where one $\boldsymbol{B}_{i}$ is designated as the set of indicators and $\boldsymbol{B}_{1} \times$ $\cdots \times \boldsymbol{B}_{m}$ is (well-) ordered in any way.

\section{§4. A variation of ordinal diagrams}

We are to study a variation of the theory $\boldsymbol{O}(\Lambda, I, \boldsymbol{A})$.

Definition 4.1. The elements of $O^{*}(\Lambda, I, A)$ are those of $O(\Lambda, I, A)$ (cf. Definition 3.1). Sections and orderings are defined as for $\boldsymbol{O}(\Lambda, I, A)$ except the order $\angle_{\infty}$ for connected elements.

Let $\tilde{\alpha}$ be $(\lambda, j, a, \alpha)$ and $\tilde{\beta}$ be $(\mu, k, b, \beta) . \tilde{\alpha} \angle_{\infty} \tilde{\beta}$ if one of the following holds.

0) $(j, a)<(k, b)$, where the order of $I \times A$ is predetermined.

In the subsequent cases, $(j, a)=(k, b)$ is assumed.

1) $\lambda=\mu$ and $\alpha \angle_{j} \beta$.

2) $\lambda<\mu$ and $\alpha<{ }_{j} \tilde{\beta}$.

3) $\lambda>\mu$ and $\tilde{\alpha} \leqslant{ }_{j} \beta$.

The following two lemmas are valid for any existing theory of ordinal diagrams and can be established prior to the linearity of the orderings.

Definition 4.2. Consider a sequence of $i$-sections in $\alpha: \alpha=\sigma_{0}, \sigma_{1}$, $\cdots, \sigma_{n}$, where $\sigma_{\ell+1}$ is an $i$-section of $\sigma_{\ell}$ and $\sigma_{n}$ is a last one. A sequence like this is called an $i$-chain of $\alpha$.

LEMma 1. For any $\alpha, \alpha=\alpha$ and only this relation holds; for any $\alpha$ and $\sigma$, where $\sigma$ is in an i-chain of $\alpha, \sigma \angle_{i} \alpha$ and only this relation holds. 
Proof. It suffices to deal with the case where everything is connected. The proof is by induction on $\ell(\alpha)$, the complexity of $\alpha$.

$0=0$ and only this relation holds.

Suppose the proposition holds for every $\beta$ where $\ell(\beta)<\ell(\alpha)$.

Let $\sigma_{0}=\alpha, \sigma_{1}, \cdots, \sigma_{n}$ be an $i$-chain of $\alpha$. To establish $\sigma_{\ell} \angle_{i} \alpha$ for every $\ell, 1 \leq \ell \leq n$, we proceed as follows. $\sigma_{\ell} L_{i} \sigma_{\ell-1}$ by definition. $\sigma_{\ell-1}$ is an $i$-section of $\sigma_{\ell-2}$, hence by definition $\sigma_{\ell} \angle_{i} \sigma_{\ell-2}$. $\sigma_{\ell-2}$ is an $i$-section of $\sigma_{\ell-3}$, hence again by definition, $\sigma_{\ell} \angle_{i} \sigma_{\ell-3}$, and so it goes until we reach $\sigma_{\ell} \angle_{i} \alpha . \quad \sigma_{\ell} \neq \alpha$ is obvious.

Suppose $\alpha \angle_{i} \sigma_{\ell}$ also holds. When $n=1, \ell=n=1$ and $\alpha \angle_{i} \sigma_{1}$ is reduced to $\alpha \angle_{j_{0}} \sigma_{1}$ for some $j_{0}>i$ and $\delta \angle_{i} \sigma_{1}$ for every $\delta$ an $i$-section of $\alpha$. $\delta$ can be $\sigma_{1}$; but $\ell\left(\sigma_{1}\right)<\ell(\alpha)$, so by the induction hypothesis $\sigma_{1} \angle_{i} \sigma_{1}$ is impossible. When $n>1$, we show that $\alpha \angle_{i} \sigma_{\ell}$ is impossible by induction on $n-\ell$.

If $\ell=n$, then $\sigma_{2}, \cdots, \sigma_{n}$ is an $i$-chain of $\sigma_{1}$ and $\ell\left(\sigma_{1}\right)<\ell(\alpha) . \quad \alpha \angle_{i} \sigma_{n}$ is reduced to the fact that $\alpha \angle_{j_{0}} \sigma_{n}$ for some $j_{0}>i$ and $\delta \angle_{i} \sigma_{n}$ for every $\delta$ an $i$-section of $\alpha$. $\delta$ can be $\sigma_{1}$; but $\sigma_{1} \angle_{i} \sigma_{n}$ is impossible by the induction hypothesis. Assume $\alpha \angle_{i} \sigma_{\ell+1}$ is impossible and suppose $\alpha \angle_{i} \sigma_{\ell}$.

Case 1. $\alpha \leqslant_{i} \sigma_{\ell+1}$. The induction hypothesis prevents this.

Case 2. $\alpha \angle{ }_{\imath} \sigma_{\ell}$ for some ${ }_{c}>i$ and $\delta \angle_{i} \sigma_{\ell}$ for every $\delta$ an $i$-section of $\alpha$. $\delta$ can be $\sigma_{1}$. But then $\sigma_{2}, \cdots, \sigma_{n}$ is an $i$-chain of $\sigma_{1}$ and $\ell\left(\sigma_{1}\right)<$ $\ell(\alpha)$, hence $\sigma_{1} \angle_{i} \sigma_{\ell}$ is impossible. (If $\ell=1, \sigma_{1} \angle_{i} \sigma_{1}$ is impossible.)

Suppose now $\alpha \angle_{i} \alpha$. We lead a contradiction by induction on $\omega \cdot \ell(\alpha)+\iota(i ; \alpha)$. Let $\alpha=(\lambda, j, b, \beta)$. If $i$ is $\infty$, then $\alpha \angle_{i} \alpha$ is reduced to $\beta \angle_{j} \beta$, contradicting the induction hypothesis. If $i$ is an indicator, then there are two cases.

Case 1. $\alpha \leqslant_{i} \sigma$ for some $\sigma$ an $i$-section of $\alpha$. But this has been crossed out.

Case 2. $\alpha \angle_{j_{0}} \alpha$ where $j_{0}=j_{0}(i ; \alpha)$ and $\sigma \angle_{i} \alpha$ for every $\sigma$ an $i$-section of $\alpha$. But $\alpha \angle_{j_{0}} \alpha$ contradicts the induction hypothesis.

LEMMA 2. Let $\alpha$ be a connected o.d. and let $\sigma$ be a j-section of $\alpha$. If $\alpha<_{j} \beta$ for $a \beta$, then $\sigma<_{j} \beta$.

Proof. By induction on $\ell(\beta)$. If $\beta$ is not connected, consider the relation for a component of $\beta$. Suppose $\beta$ is connected.

Case 1. $\alpha \leqslant_{j} \delta$ for a $\delta$ a $j$-section of $\beta$. Then $\sigma L_{j} \delta$ either by Lemma 1 or by the induction hypothesis. So by definition $\sigma \angle_{j} \beta$. 
Case 2. $\alpha \angle_{j_{0}} \beta$ for some $j_{0}>j$ and $\eta \angle_{j} \beta$ for every $\eta$ a $j$-section of $\alpha$. Let $\eta$ be $\sigma$.

Proposition 4.1. $\boldsymbol{O}^{*}(\Lambda, \boldsymbol{I}, \boldsymbol{A})$ is linearly ordered for every $\angle_{i}$.

Proof. The proof of Proposition 26.8 in [7] goes through except for the case $i$ is $\infty$, which will be dealt with here. Let $\tilde{\alpha}=(\lambda, j, a, \alpha)$, $\tilde{\beta}=(\mu, k, b, \beta)$ and $\tilde{\gamma}=(\nu, m, c, \gamma)$.

I. Suppose $\tilde{\alpha} \angle_{\infty} \tilde{\beta}$ and $\tilde{\beta} \angle_{\infty} \tilde{\gamma}$. If $(j, a)<(m, c)$, then $\tilde{\alpha} \angle_{\infty} \tilde{\gamma}$ by 0$)$ of Definition 4.1. So, let us assume $(j, a)=(k, b)=(m, c)$.

Case 1. $\lambda=\mu=\nu . \quad \alpha{ }_{j} \beta$ and $\beta \angle_{j} \gamma$. By the induction hypothesis $\alpha L_{j} \gamma$, and hence $\tilde{\alpha} \angle_{\infty} \tilde{\gamma}$ by 1 ).

Case 2. $\lambda=\mu<\nu . \quad \alpha{ }_{j} \beta$ and $\beta \angle_{j} \tilde{\gamma}$. By the induction hypothesis $\alpha{ }_{j} \tilde{\gamma}$, hence $\tilde{\alpha} \angle_{\infty} \tilde{\beta}$ by 2 ).

Case 3. $\lambda=\mu>\nu$. $\alpha \angle_{j} \beta$ and $\tilde{\beta} \leqslant{ }_{j} \gamma$. It suffices to show that $\tilde{\alpha} \angle{ }_{j} \tilde{\beta}$. But $\alpha{ }_{j} \beta$ implies $\alpha{ }_{j}, \tilde{\beta}$, for $\beta$ is a $j$-section of $\tilde{\beta}$, and $\alpha{ }_{j} \tilde{\beta}$ and $\tilde{\alpha} \angle_{\infty} \tilde{\beta}$ imply $\tilde{\alpha} \angle_{j} \tilde{\beta}$, for $\alpha$ is the $j$-section of $\tilde{\alpha}$.

Case 4. $\lambda<\mu=\nu$. $\alpha \angle, \tilde{\beta}$ and $\beta \angle{ }_{j} \gamma$. It suffices to show that $\tilde{\beta} \angle_{j} \tilde{\gamma}$. But, since $\tilde{\beta} \angle_{\infty} \tilde{\gamma}$ and $\beta$ is the $j$-section of $\tilde{\beta}, \beta L_{j} \tilde{\gamma}$ will imply the desired relation. $\beta L_{j} \tilde{\gamma}$ follows from $\beta L_{j} \gamma$ and the fact that $\gamma$ is $j$-section of $\tilde{\gamma}$.

Case 5. $\lambda<\mu<\nu . \quad \alpha \angle{ }_{j} \tilde{\beta}$ and $\beta \angle_{j} \tilde{\gamma}$. It suffices to show that $\tilde{\beta} \angle_{j} \tilde{\gamma}$. But $\tilde{\beta} \angle_{\infty} \tilde{\gamma}$ and $\beta \angle_{j} \tilde{\gamma}$ imply that $\tilde{\beta} \angle_{j} \tilde{\gamma}$.

Case 6. $\lambda<\mu$ and $\mu>\nu . \quad \alpha{ }_{j} \tilde{\beta}$ and $\tilde{\beta} \leqslant{ }_{j} \gamma . \quad \alpha{ }_{j} \gamma$ by the induction hypothesis.

Case 6.1. $\lambda=\nu . \quad \alpha L_{\gamma} \gamma$ implies $\tilde{\alpha} \angle_{\infty} \tilde{\gamma}$ by 1 ).

Case 6.2. $\lambda<\nu$. For each component of $\alpha$, say $\sigma, \sigma \angle{ }_{j} \gamma$, hence $\sigma{ }_{j} \tilde{\gamma}$. Thus $\alpha{ }_{1} \tilde{\gamma}$, hence $\alpha \angle_{\infty} \beta$ by 2).

Case 6.3. $\lambda>\nu$. It needs be shown $\tilde{\alpha} \angle_{j} \tilde{\beta}$. But $\tilde{\alpha} \angle_{\infty} \tilde{\beta}$ and $\alpha \angle_{j} \tilde{\beta}$ where $\alpha$ is the $j$-section of $\tilde{\alpha}$, so $\tilde{\alpha} \angle{ }_{j} \tilde{\beta}$.

Case 7. $\lambda>\mu=\nu . \quad \tilde{\alpha} \leqslant_{j} \beta$ and $\beta \angle_{j} \gamma$. By the induction hypothesis $\tilde{\alpha} \angle_{j} \gamma$, hence $\tilde{\alpha} \angle_{\infty} \tilde{\gamma}$ by 3).

Case 8. $\quad \lambda>\mu>\nu . \quad \tilde{\alpha} \leqslant_{j} \beta$ and $\tilde{\beta} \leqslant_{j} \gamma . \quad \tilde{\alpha} \angle_{j} \tilde{\beta}$, hence $\tilde{\alpha} \angle_{j} \gamma$ by the induction hypothesis, which implies $\tilde{\alpha} \angle_{\infty} \tilde{\gamma}$.

Case 9. $\lambda>\mu$ and $\mu<\nu . \quad \tilde{\alpha} \angle_{j} \beta$ and $\beta \angle_{j} \tilde{\gamma} . \quad \tilde{\alpha} \angle_{j} \tilde{\gamma}$ by the induction hypothesis.

Case 9.1. $\lambda=\nu$. $\quad \tilde{\alpha} \angle_{j} \tilde{\gamma}$. If this is so because $\tilde{\alpha} L_{j} \gamma$ ( $\gamma$ is a $j$ section of $\tilde{\gamma}$ ), then by Lemmas 1 and $2 \alpha L_{j} \gamma$ ( $\alpha$ is a $j$-section of $\tilde{\alpha}$ ), 
hence $\tilde{\alpha} \angle_{\infty} \tilde{\gamma}$. If $\tilde{\alpha} \angle_{j} \tilde{\gamma}$ because $\tilde{\alpha} \angle_{\infty} \tilde{\gamma}$ and $\alpha \angle_{j} \tilde{\gamma}$, then $\tilde{\alpha} \angle_{\infty} \tilde{\gamma}$ by the assumption.

Case 9.2. $\lambda<\nu$. From $\tilde{\alpha} \angle_{j} \tilde{\gamma}$ follows $\alpha \angle_{j} \tilde{\gamma}$ by Lemma 2, so $\tilde{\alpha} \angle_{\infty} \tilde{\gamma}$.

Case 9.3. $\lambda>\nu$. If $\tilde{\alpha} \angle_{j} \tilde{\gamma}$ because $\tilde{\alpha} \leqslant_{j} \gamma$, then $\tilde{\alpha} \angle_{\infty} \tilde{\gamma}$ by 3). If this is so because $\tilde{\alpha} \angle_{\infty} \tilde{\gamma}$ and $\alpha \angle_{j} \tilde{\gamma}$, then $\tilde{\alpha} \angle_{\infty} \tilde{\gamma}$ by the assumption.

III. Suppose $\tilde{\alpha} \neq \tilde{\beta}$. If $(j, a)>(k, b)$ or $(j, a)<(k, b)$, then $\tilde{\alpha}_{\infty}>\tilde{\beta}$ or $\tilde{\alpha} \angle_{\infty} \tilde{\beta}$ accordingly. Suppose $(j, a)=(k, b)$. If $\lambda=\mu$, then $\alpha \angle_{j} \beta$ or $\beta \angle_{j} \alpha$ and only one of those holds, hence exactly one of $\tilde{\alpha} \angle_{\infty} \tilde{\beta}$ or $\tilde{\beta} \angle_{\infty} \tilde{\alpha}$. Suppose $\lambda<\mu$. $\alpha \angle_{j} \tilde{\beta}$ or $\tilde{\beta} \leqslant_{j} \alpha$ by the induction hypothesis. So $\tilde{\alpha} \angle_{\infty} \tilde{\beta}$ by 2 ) or $\tilde{\beta} \angle_{\infty} \tilde{\alpha}$ by 3 ) as the case may be.

Proposition 4.2. Let $\tilde{\alpha}$ and $\tilde{\beta}$ be connected elements of $\boldsymbol{O}^{*}(\Lambda, \boldsymbol{I}, \boldsymbol{A})$ which share a same outermost value, say $(i, a)$. Then $\tilde{\alpha} \angle_{i} \tilde{\beta}$ implies $\tilde{\alpha} \angle_{p} \tilde{\beta}$ for every $p \geq i$.

Proof. Let $\tilde{\alpha}$ be $(\lambda, i, a, \alpha)$ and let $\tilde{\beta}$ be $(\mu, i, a, \beta)$. Assume $\tilde{\alpha} \angle_{i} \tilde{\beta}$ and suppose $\tilde{\beta} \angle_{\infty} \tilde{\alpha}$. Then

$$
\tilde{\alpha} \leq i \beta
$$

There are three cases: $\mu=\lambda$ and $\beta \angle_{i} \alpha ; \mu<\lambda$ and $\beta \angle_{i} \tilde{\alpha} ; \lambda<\mu$ and $\tilde{\beta} \leqslant_{i} \alpha$. In any case, $\beta \angle_{i} \tilde{\alpha}$ follows, contradicting (*). Thus $\tilde{\alpha} \angle_{\infty} \tilde{\beta}$ must be the case, hence $\tilde{\alpha} \angle_{p} \tilde{\beta}$ for every $p \geq i$.

THEOREM 4. $\boldsymbol{O}^{*}(\Lambda, \boldsymbol{I}, \boldsymbol{A})$ is well-ordered by $\angle_{i}$ for every $i$ in $\boldsymbol{I} \cup\{\infty\}$.

Proof. The proof of Theorem 26. 14 in [7] almost goes through. Only Lemma 26. 28 requires some supplement.

LEMMA 26. 28*. Every element of $\boldsymbol{F}_{\infty}$ is $\angle_{\infty}$-accessible in $F_{\infty}$.

Proof. Suppose not, that is, suppose there is a sequence $\left\{\tilde{\alpha}_{n}\right\}_{n}$ of elements of $\boldsymbol{F}_{\infty}$ which is strictly decreasing with respect to $\angle_{\infty}$. We may assume that $\alpha_{n}$ 's are connected. Recall that for $\angle_{\infty}$ those elements are first compared by their outermost values $(i, a)$. Since those values are well-ordered, a decreasing sequence of values must be finite. Thus, after a certain stage, the outermost values of $\tilde{\alpha}_{n}$ will be constant, say $(j, b)$. To make the discussion simple, we assume this is the case for every $n, n=1,2, \ldots$. So $\tilde{\alpha}_{n}$ has the form $\left(\lambda_{n}, j, b, \alpha_{n}\right)$. Since $\Lambda$ is wellordered, there is an increasing sequence of subscripts, $\left\{i_{n}\right\}_{n}$, such that $\lambda_{i_{m}} \leq \lambda_{i_{m+1}}$ for every $m$. If $=$ holds for infinitely many $m$ 's, we may 
assume that it is the case for all $m$, thus $\alpha_{i_{m+1}} L_{j} \alpha_{i_{m}}$ for all $m$. If $j$ is the maximal element in $I$, then $\tilde{\alpha}_{i_{m}} \in F_{\infty}$ means that every $j$-section of it is $\angle_{j}$-accessible in $F_{j}$. If $j$ is not the maximal element of $I$, then $\tilde{\alpha}_{i_{m}} \in \boldsymbol{F}_{j+1}$ and hence every $j$-section of it is $\angle_{j}$-accessible in $\boldsymbol{F}_{j}$. In either case, there cannot be an infinite decreasing sequence of such $j$ sections, hence $\left\{\tilde{\alpha}_{i_{m}}\right\}_{m}$ cannot exist. Suppose next $\angle$ holds for infinitely many $m$ 's. We may assume this is so for all $m$. Then $\tilde{\alpha}_{i_{m+1}} \leqslant{ }_{j} \alpha_{i_{m}}$ (cf. 3) of Definition 4.1), hence $\alpha_{i_{m+1}} L_{j} \alpha_{i_{m}}$. With the same reasoning as above, we are led to a contradiction. Thus follows the accessibility of $\boldsymbol{F}_{\infty}$ with regards to $\angle_{\infty}$.

THEOREM 5. The theory of approximations can be developed for $\boldsymbol{O}^{*}(\Lambda, \boldsymbol{I}, \boldsymbol{A})$.

The proof is similar to that of Theorem 3, $\S 3$. Here we return to the original value, however: a value is an element of $I \times A$. We shall only point out a few aspects which did not arise in $\S 3$.

Lemma 26. $45^{*}$. (2) $\delta \angle_{i} \gamma$ does not necessarily hold in $O^{*}(\Lambda, I, A)$, but $\alpha^{\prime} \angle_{m} \alpha_{0}, m \geq i$, does hold, by virtue of Proposition 4.2.

(4) (ii) $(k, b)=(i, a)$. $\eta \angle_{m} \alpha_{0}, m \geq i$, by (2) above.

(5) is not guaranteed as was remarked in $\S 3$.

Proposition 26. 46*. (2) in the proof follows from Proposition 4.2.

Proposition 26. 47*. Read as in $\S 3$.

The rest of the material in $\S 3$ goes through.

A remark on fundamental sequences.

Fundamental sequences can be constructed uniformly both for $\boldsymbol{O}(\Lambda, \boldsymbol{I}, \boldsymbol{A})$ and $\boldsymbol{O}^{*}(\Lambda, \boldsymbol{I}, \boldsymbol{A})$. We do not elaborate on this matter now, since, a routine work though it may be, it takes enormous time and space to actually execute the construction. It will not be too late if we take it up when one of those systems is applied to a consistency proof. (See [8] and [9] for the detail.)

\section{REFERENCES}

[1] G. Takeuti, Ordinal diagrams, J. Math. Soc. Japan, 9 (1957), 386-394.

[2] - Ordinal diagrams II, J. Math. Soc. Japan, 12 (1960), 385-391.

[ 3 ] — Consistency proofs of subsystems of classical analysis, Annals of Mathematics 86 (1967), 299-348. 
[4] - The $\Pi_{1}^{1}$-comprehension schema and $\omega$-rules, Proceedings of the Summer School in Logic (1967), Lecture Notes in Mathematics, 70 (1968), 303-330.

[5] M. Yasugi, Cut elimination theorem for second order arithmetic with the $\Pi_{1}^{1-}$ comprehension axiom and the $\omega$-rule, J. Math. Soc. Japan, 22 (1970), 308-324.

[6] G. Takeuti and M. Yasugi, The ordinals of the systems of second order arithmetic with the provably $\Delta_{2}^{1}$-comprehension axiom and with the $\Delta_{2}^{1}$-comprehension axiom respectively, Japanese J. of Math., 41 (1973), 1-67.

[ 7 ] G. Takeuti, Proof Theory, North-Holland Publ. Co., Amsterdam, 1975.

[ 8 ] G. Takeuti and M. Yasugi, Fundamental sequences of ordinal diagrams, Comentariorum Mathematicorum Universitatis Sancti Pauli, 25 (1976), 1-80.

[9] — An accessibility proof of ordinal diagrams, Mimeographed Notes.

Shizuoka University 